\title{
Outcome of Microsurgical Revascularization in Patients with Moyamoya Disease: First Report from the Iranian Population
}

\author{
Abdolkarim RAHMANIAN ${ }^{1}$, Mohammad Sadehgh MASOUDI ${ }^{1}$, Fariborz GHAFFARPASAND ${ }^{2}$, \\ Mohammad Hossein ASHRAF $^{2}$, Ehsanali ALIBAI ${ }^{1}$ \\ ${ }^{1}$ Shiraz University of Medical Sciences, Department of Neurosurgery, Fars, Iran \\ ${ }^{2}$ Shiraz University of Medical Sciences, Student Research Committee, Department of Neurosurgery, Fars, Iran
}

\section{ABSTRACT}

AIM: To report the outcome of microsurgical revascularization in patients with Moyamoya Disease (MMD) in Southern Iran.

MATERIAL and METHODS: This cross-sectional study was conducted in Southern Iran during a 7-year period from 2009 to 2016. All the patients with Moyamoya Disease (confirmed with digital substraction angiography) who underwent microsurgical revascularization (extracranial-intracranial bypass or synangiosis) were included. All the patients were followed for at least 1 year and the outcome was measured using the Glasgow outcome scale (GOS) and modified Rankin scale (MRS).

RESULTS: Overall we included 13 patients with 14 involved hemispheres undergoing direct and indirect revascularization. The mean age of the patients was $20.6 \pm 17.5$ (ranging from 0.5 to 55 ) years and there were $5(38.4 \%)$ males and $8(61.6 \%)$ females. We did not have any unfavorable outcome defined as mortality and persistent vegetative state. We performed 12 (85.7\%) superficial temporal artery (STA)-middle cerebral artery (MCA) bypass and 2 (14.3\%) encephalo-myo-synangiosis (EMS) procedures. The symptoms improved in 7 (53.8\%) patients and remained as the preoperative course in 5 (38.5\%) patients. Only 1 (7.7\%) patient developed immediate postoperative vasospasm and brain swelling, and was managed successfully with decompressive craniectomy and subsequent cranioplasty (GOS $=5$, MRS $=0$ ).

CONCLUSION: This is the first study to report the safety, efficacy and outcome of the direct (STA-MCA bypass) and indirect (EMS) revascularization in patients with MMD in the Iranian population. As the prevalence of MMD is low in Iranian population, the experience and technique remains in its infancy and further advancements in the field is required.

KEYWORDS: Extracranial-intracranial bypass, Iran, Moyamoya Disease, Revascularization, Synangiosis

\section{INTRODUCTION}

$\mathrm{M}$ oyamoya Disease (MMD) is a chronic, progressive and idiopathic stenosis and occlusion of intracranial segments of the internal carotid artery (ICA) leading to cerebral hypoperfusion and ischemia followed by subsequent formation of collateral abnormal intracranial arteries $(8,17)$. The angiographic characteristics of bilateral ICA was first described by Takeuchi and Shimizu in 1957 (30), while the MMD (something hazy like puff of smoke) term was proposed in 1969 for these patients (29). Currently, MMD has been reported and described from all over the world and different ethnic groups (17), but the disease remains the most common cerebrovascular disease of children in Southeast Asian countries, especially Japan and China $(3,21)$. It is considered the cause of $6 \%$ of all ischemic strokes in the pediatric population and the prevalence is increasing, probably due to increased awareness and diagnosis of the condition $(3,12,17)$. In European countries, the incidence of MMD has been reported to be 0.3 patients per center per year which is one-tenth of the incidence reported in Japan $(18,31)$. The disease has been reported rarely in Iran, and there is no data available on the natural course, outcome and surgical management of these patients from Iran $(2,16,27)$. 
The treatment of MMD focuses on preventing cerebral ischemia and stroke by improving blood flow to the affected cerebral hemispheres $(4,20)$. None of the available treatment options alter the natural course and the final outcome of patients with MMD, but only provide an alternative blood supply for the affected hemispheres to prevent further neurological deficits and disability $(5,11,32)$. There is no medical therapy available for the disease and surgical revascularization remains the mainstay of the treatment $(15,22,28)$. The revascularization surgery includes both direct superficial temporal artery (STA)-middle cerebral artery (MCA) anastomosis and indirect revascularization using encephalomyo-synangiosis (EMS) $(1,10,14,15,25)$. There is lack of data from the Iranian population regarding the natural course and outcome of patients with MMD.

In the current study, we report the clinical and outcome characteristics of first series of MMD from Iran, consisting of patients undergoing revascularization surgery with both direct and indirect techniques.

\section{MATERIAL and METHODS}

\section{Study Population}

This cross-sectional study was conducted in a 7-year period from 2009 to 2016 in Namazi Hospital, a tertiary healthcare center and the main cerebrovascular referral center in Southern Iran affiliated with Shiraz University of Medical Sciences. The study protocol was approved by the institutional review board and the medical ethics committee of Shiraz University of Medical Sciences and all the patients. As this was a retrospective review of the medical charts, informed written consent was not required and the patients' information remained secure. We included all the patients with confirmed MMD who underwent surgical revascularization in our center during the study period. The diagnosis of MMD was confirmed according to the guidelines published by the Research Committee on MMD (spontaneous occlusion of the circle of Willis) of Japan (26). Accordingly, those with cranial irradiation, meningitis, Down syndrome, Von Recklinghausen syndrome, intracranial tumors and previous cranial surgery were excluded from the study. In pediatric patients, we considered unilateral Moyamoya vasculopathy as the definite MMD if steno-occlusion was identified on another side of the ICA terminal (26).

\section{Study Protocol}

The medical charts of those that were found to be eligible to be included in the study were extracted and studied. The demographic information, presenting symptoms, imaging findings and the operation type were recorded in data gathering forms and were entered into a computer database. All the patients were called for outpatient visits. The patients were seen at the clinic at least 12 months after the operation. The clinical outcome was evaluated using Glasgow Outcome Scale (GOS) and modified Rankin Score (MRS). We also recorded the improvement in the initial symptoms and progression of steno-occlusion symptoms. Postoperative stroke or transient ischemic attack (TIA) was defined as a new focal neurologic deficit associated with imaging findings in magnetic resonance imaging (MRI) or brain computed tomography (CT) scan (Figure 1A). All the patients had preoperative digital subtraction angiography (DSA) of both carotid arteries which was studies again (Figures 1B, C).

\section{Surgical Technique}

All the patients were operated at the same center by the same surgeon (first author) using the same protocol. The surgical revascularization technique included direct (STA-MCA bypass) and indirect (EMS) methods. All the patients were operated under general anesthesia with specific consideration in MMD $(11,15)$. We used STA-MCA bypass in all the patients undergoing direct revascularization. In those undergoing indirect revascularization, STA was also used for EMS. In brief, the patient was placed in the supine position with the head fixed in a Sugita or Mayfield head frame or horseshoe, and then rotated to the contralateral side for 45 degrees and extended for 10 degrees. An incision was made over the STA as described elsewhere and the frontal and parietal branches of the STA were dissected and exposed completely under the microscope. Then, the temporalis muscle was divided and a craniotomy was performed in temporoparietal region over the Sylvian fissure. We opened the dura in C-shape manner with the base over the superior sagittal sinus and with specific caution to save the branches of the middle meningeal artery. The dura was then reflected into the subdural space in order to produce the encephalo-duro-synangiosis (EDS). After inspecting the cerebral cortex, an appropriate M4 branch was identified and prepared as the recipient artery. A triangular silicon background was applied and two non-traumatic temporary clips were placed at the proximal and distal parts of the M4 branch (Figure 1D). Another temporary clip was applied at the proximal part of the STA and the distal part was cut in a fishmouth pattern. Methylene blue was applied on the anastomosis site (both donor and recipient arteries) in order to facilitate the visualization of the vessels. A linear arteriotomy was performed in the side of M4 branch and the anastomosis was performed under a high power microscope using round, non-cutting 10.0 polypropylene sutures (PROLENE® Polypropylene Suture, Ethicon Inc., New Jersey, USA). About 8-10 interrupted sutures were applied to each anastomosis (Figure 1E). The proximal and distal temporary clips were removed respectively to assess the patency of the graft. Then the proximal STA clip was finally removed. The patency of the graft was checked by intraoperative Doppler ultrasonography (Transonic Systems Inc., Ithaca NY) and indocyanine green video angiography (OPMI® Pentero, Carl Zeiss Co., Oberkochen, Germany) (Figure 1F). The EMS was obtained by covering the cortex with the temporalis muscle and securing it by suturing it to the dural edges. Cranioplasty was performed on the temporalis muscle using a flap fix and an opening was performed in the temporal floor for the STA graft. No compression was applied on the graft and the patient was instructed not to lie on the graft side and not to apply any pressure on the side of the graft. All the patients received intraoperative infusion of Nimodipine (Nimotop $®$, Injection 0.2 $\mathrm{mg} / \mathrm{mL}$, Bayer Schering Pharma, Berlin, Germany) at a dose 
of $0.35 \mu \mathrm{g} / \mathrm{kg} / \mathrm{min}$ during the operation and this was continued for 3 postoperative days in order to prevent vasospasm. The patients also received $100 \mathrm{mg}$ oral Aspirin daily from the first postoperative day for 3 months.

\section{Statistical Analysis}

All the statistical analysis was performed using the Statistical Package for Social Sciences (SPSS Inc., Chicago, Illinois, USA) version 21.0. Data are presented as mean \pm SD and proportions as appropriate and are summarized in tables.

\section{- RESULTS}

We included a total of 13 patients with 14 involved hemispheres undergoing direct and indirect revascularization at our center during the study period. Only $3(23.1 \%)$ adults were included while the others were pediatric patients $(76.9 \%)$. The mean age of the patients was $20.6 \pm 17.5$ (ranging from 0.5 to 55 ) years. There were $5(38.4 \%)$ males and $8(61.6 \%)$ females. Only $3(23.1 \%)$ patients had a positive family history and 1 $(7.7 \%)$ had sickle cell anemia. Ischemic infarction and TIA were among the most common presenting symptoms while behavioral changes and failure to thrive in a 6-month-old baby were the least common ones. In 12 (85.7\%) patients we performed STA-MCA bypass along with EMS while in 2 (14.3\%) the STA-MCA bypass could not be performed as we could not find an appropriate M4 branch. The demographic and baseline characteristics are summarized in Table I.

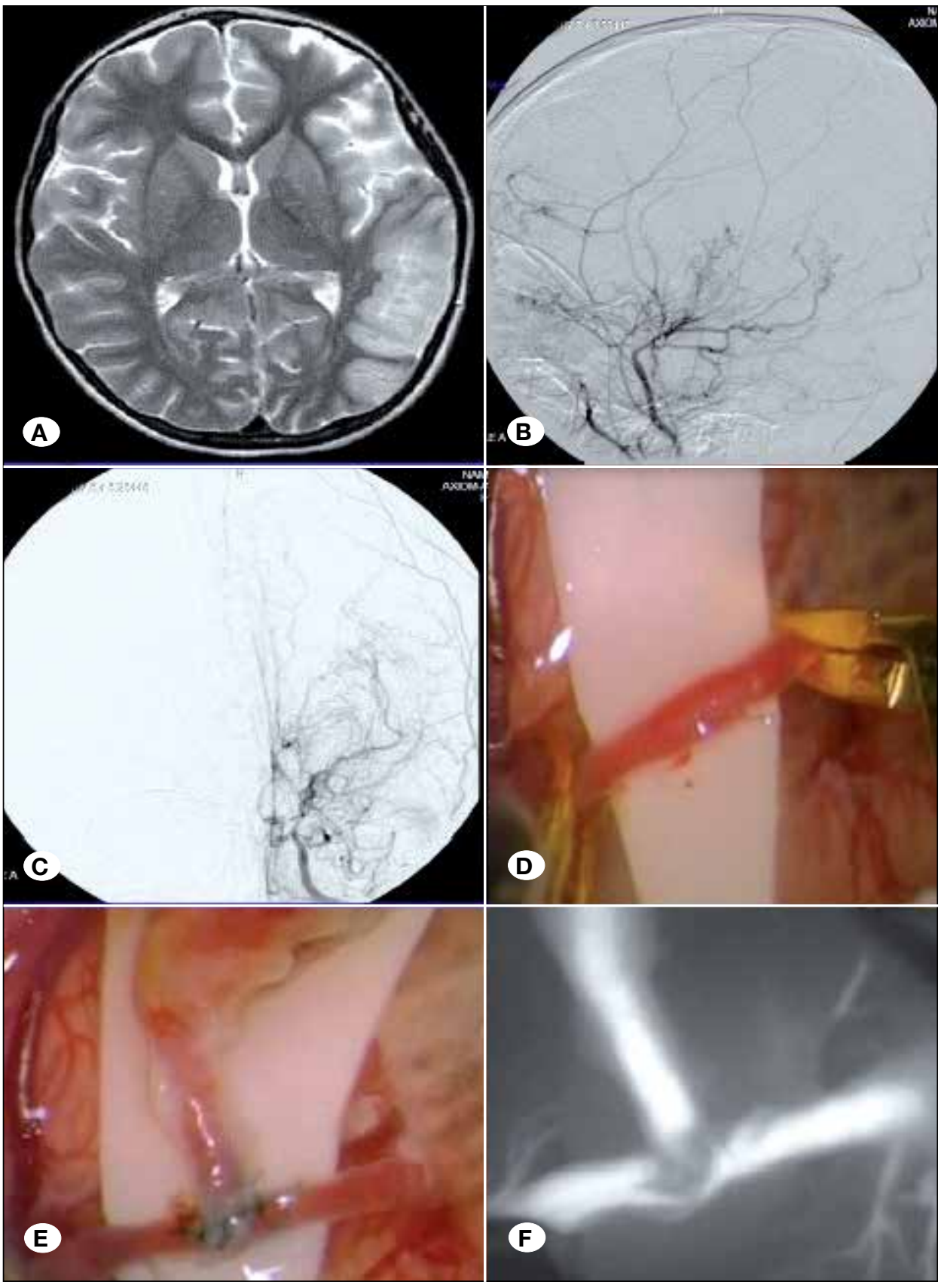

Figure 1: A 5-year-old girl presented with right sided weakness and 2 episodes of tonic-clonic seizure. The T2-weighted MRI demonstrated ischemic changes of left cortical regions (A); digital subtraction angiography of left carotid artery in sagittal (B) and coronal (C) views demonstrated the steno-occlusion changes of distal branches and development of collateral arteries (puff of smoke appearance). The surgical procedure of superficial temporal artery (STA)-middle cerebral artery (MCA) anastomosis: detection of appropriate M4 branch and clipping it bilaterally (D); end to side anastomosis of STA-MCA (M4 branch) (E); and the intraoperative ICG angiography demonstrating the patency and adequacy of the graft (F). 
The intraoperative characteristics and the outcome measures of the patients are summarized in Table II. In 6 patients, one M4 graft was used and in the other 6 patients two grafts were placed. We did not have any unfavorable outcome that was defined as mortality and persistent vegetative state (PVS). The symptoms improved in 7 (53.8\%) patients and these patients did not develop further symptoms. The symptoms remained as in the preoperative course in 5 (38.5\%) patients. Only 1 (7.7\%) patient developed immediate postoperative brain swelling and vasospasm which was managed by decompressive craniectomy and subsequent cranioplasty resulting in complete recovery $(\mathrm{GOS}=5$; MRS $=0)$. Only $1(7.7 \%)$ patient underwent bilateral STA-MCA bypass grafting and the other did not develop symptoms in the contralateral side.

Table I: The Baseline Characteristics of 13 Patients and 14 Involved Hemispheres with Confirmed Moyamoya Disease Undergoing Direct and Indirect Revascularization at Our Center

\begin{tabular}{|c|c|}
\hline Variable & Value \\
\hline Age (years) & $20.6 \pm 17.5$ \\
\hline \multicolumn{2}{|l|}{ Age category } \\
\hline Pediatric patients (\%) & $10(76.9 \%)$ \\
\hline Adults (\%) & $3(23.1 \%)$ \\
\hline \multicolumn{2}{|l|}{ Gender } \\
\hline Male (\%) & 5 (38.4\%) \\
\hline Female (\%) & $8(61.6 \%)$ \\
\hline Family history (\%) & $3(23.1 \%)$ \\
\hline \multicolumn{2}{|l|}{ Co-morbidities } \\
\hline Hypertension (\%) & $2(15.4 \%)$ \\
\hline Sickle cell anemia (\%) & $1(7.7 \%)$ \\
\hline \multicolumn{2}{|l|}{ Presenting Symptoms } \\
\hline Infarction (\%) & $7(53.8 \%)$ \\
\hline Transient ischemic attack (\%) & $3(23.1 \%)$ \\
\hline Intraparenchymal hemorrhage (\%) & $3(23.1 \%)$ \\
\hline Seizures (\%) & $2(15.4 \%)$ \\
\hline Cognitive impairment (\%) & $2(15.4 \%)$ \\
\hline Dysphasia (\%) & $2(15.4 \%)$ \\
\hline Failure to thrive (\%) & $1(7.7 \%)$ \\
\hline Behavioral changes (\%) & $1(7.7 \%)$ \\
\hline Visual field defect (\%) & $1(7.7 \%)$ \\
\hline \multicolumn{2}{|l|}{ Operation } \\
\hline STA-MCA bypass (\%) & $12(85.7 \%)$ \\
\hline EMS (\%) & $2(14.3 \%)$ \\
\hline \multicolumn{2}{|l|}{ Side } \\
\hline Right (\%) & 9 (69.2\%) \\
\hline Left (\%) & $3(23.1 \%)$ \\
\hline Bilateral (\%) & $1(7.7 \%)$ \\
\hline
\end{tabular}

EMS: Encephalo-myo-synangiosis, MCA: Middle cerebral artery, STA: Superficial temporal artery.

\section{DISCUSSION}

MMD is a chronic progressive disease of the internal carotid disease resulting in cerebral hypoperfusion and long term neurologic deficits (8). Although it has been described in Southeast Asian populations, reports from other ethnicities exist and the prevalence is increasing due to improvements in diagnosis $(3,11,17)$. Currently, it has been demonstrated that revascularization surgery is the only effective treatment in these patients (19). This small series, to the best of our knowledge, is considered the first report from the Iranian population in the literature. We report our experience and outcome of 13 Iranian patients (14 hemispheres) with confirmed MMD who underwent revascularization surgery (direct and indirect) during a 7-year period. We demonstrated that surgery resulted in improved symptoms and avoidance of further ischemic events in most of the patients. We experienced no unfavorable

Table II: The Intraoperative Characteristics and Outcome of 13 Patients and 14 Involved Hemispheres with Confirmed Moyamoya Disease Undergoing Direct and Indirect Revascularization at Our Center

\begin{tabular}{lc}
\hline Variable & Value \\
\hline Number of grafts & $1.21 \pm 0.8$ \\
\hline $0(\%)$ & $2(14.4 \%)$ \\
\hline $1(\%)$ & $6(42.8 \%)$ \\
\hline $2(\%)$ & $6(42.8 \%)$ \\
\hline Operation duration (hours) & $5.2 \pm 2.6$ \\
\hline Intraoperative bleeding (mL) & $186.4 \pm 99.8$ \\
\hline Follow-up duration (months) & $46.6 \pm 23.1$ \\
\hline 6 -month Glasgow Outcome Scale & \\
\hline Good recovery (\%) & $8(61.5 \%)$ \\
\hline Moderate disability (\%) & $5(38.5 \%)$ \\
\hline Severe disability (\%) & $0(0.0 \%)$ \\
\hline Persistent vegetative state (\%) & $0(0.0 \%)$ \\
\hline Death (\%) & $0(0.0 \%)$ \\
\hline 6-month Modified Rankin Scale & \\
\hline No symptoms (\%) & $6(46.2 \%)$ \\
\hline No significant disability (\%) & $2(15.3 \%)$ \\
\hline Slight disability (\%) & $5(38.5 \%)$ \\
\hline Moderate disability (\%) & $0(0.0 \%)$ \\
\hline Moderately severe disability (\%) & $0(0.0 \%)$ \\
\hline Severe disability (\%) & $0(0.0 \%)$ \\
\hline Death (\%) & $0(0.0 \%)$ \\
\hline
\end{tabular}

EMS: Encephalo-myo-synangiosis, MCA: Middle cerebral artery, STA: Superficial temporal artery. 
outcome (mortality and PVS) and could effectively follow and treat the complications.

Recently, a large cohort study reported the outcome of surgical revascularization in MMD patients from China (20). Liu et al.(20) reported the outcome of patients with MMD during a 25 -year period including 528 patients. They demonstrated that patients with hemorrhagic MMD had a much higher rate of re-bleeding and poorer prognosis than those with the ischemic type. Surgical revascularization was demonstrated to improve cerebral perfusion and prevent the re-bleeding in patients with hemorrhagic MMD effectively (20). Esposito et al.(7) reported their results of STA-MCA and EMS in 8 pediatric patients with MMD. They demonstrated that all the patients had an improvement in their symptoms and did not develop further deficits (7). The results of the revascularization procedures have also been described in adults with $\operatorname{MMD}(6,11,28)$. Starke et al.(28) reported their experience with encephaloduroarteriosynangiosis (EDAS) in 43 adult patients (mostly Caucasian in North America) with MMD. They demonstrated that indirect bypass promoted adequate pial collateral development and increased perfusion in the majority of the patients. The patients had low rates of postoperative TIAs, infarction, and hemorrhage, and the majority of patients had preserved or improved functional status (28). In the current series, we included both pediatric patients and adults in whom both STA-MCA bypass grafting and EMS were performed. The outcome was favorable in all the patients and no further TIA and ischemic or hemorrhagic events occurred. One of our patients developed immediate postoperative vasospasm and brain swelling which was successfully managed by decompressive craniectomy and her neurological status was improved $(\mathrm{GOS}=5, \mathrm{MRS}=0)(25)$. These complications have been previously described in larger series $(6,20,22,32)$.

Among our series, 3 (23.1\%) were hemorrhagic and others (76.9\%) were the ischemic type. None of those 3 patients with primary hemorrhagic presentation experienced postoperative hemorrhagic event during the mean 38.6 months of follow-up. This finding demonstrates that surgery is effective in reducing the risk of hemorrhage in hemorrhagic types of MMD. A recent meta-analysis demonstrated that revascularization surgery is associated with greater reduction in re-bleeding attack and ischemic stroke resulting in significant morbidity and mortality and need for further revascularization surgery compared with non-surgical therapy (19). Our findings are consistent with the aforementioned study. In another study, Fujimura et al.(9) demonstrated that STA-MCA anastomosis is a safe and effective treatment of MMD. They identified the adult-onset and/or hemorrhagic-onset patients as high risk group for symptomatic hyperperfusion after surgery, resulting in parenchymal hemorrhage and worse prognosis. Thus, they recommend routine cerebral blood flow (CBF) measurement in the high risk group to predict the adverse events (9). We did not measure the CBF due to lack of appropriate facilities, but followed and assessed the patients clinically according to the standard guidelines (26).
We note some important limitations to our study. First, this was a small series with limited number of included patients. Accordingly, we could not analyze our database in order to determine the prognostic factors and predictive factors of the outcome in this population. This point should be taken into consideration that our center is the main referral center for cerebrovascular diseases in southern Iran $(23,24)$ and thus this small series might be the largest one in Iran. Second, we did not evaluate the genetic background of the patients. It has been demonstrated that some genetic factors such as RNF213 are attributable to the pathogenesis of the disease (13). This test is not available at our center and thus we cannot comment on the issue although 3 patients had a positive family history of the disease. And, finally, we did not measure the cerebral perfusion postoperatively using different methods such as Xenon-enhanced CT-perfusion scan or $\mathrm{H}_{2} \mathrm{O}$ positron emission tomography (PET) scan. These modalities are appropriate for assessing the cerebral perfusion, but the clinical outcome has been recommended as the preferred method of assessment. Taking all these together, this is the first report of revascularization surgery in Iranian patients with MMD and the subject requires further attention and investigations.

\section{CONCLUSION}

This is the first study to report the safety, efficacy and outcome of the direct (STA-MCA bypass) and indirect (EMS) revascularization in patients with MMD in the Iranian population. As the prevalence of MMD is low in the Iranian population, the experience and technique remains in its infancy and further advancements in the field are required.

\section{ACKNOWLEDGMENT}

The authors would like to acknowledge all the patients and their families who participated in the current study. We would also like to thank the operating room technicians, anesthesiology team and the intensive care unit staff, without whom this setting could not be established. We would also like to acknowledge the editorial assistance of Diba Negar Research Institute for improving the style and English of the manuscript.

\section{REFERENCES}

1. Abdulrauf SI: Cerebral revascularization: Techniques in extracranial-to-intracranial bypass surgery: Expert Consult: Elsevier Health Sciences, 2010

2. Ashrafi MR, Alizadeh H, Yazdani SH, Mohseni M, Mohamadi M: Psychomotor delay, a possible rare presentation of moyamoya disease. Iran J Radiol 8:75-78, 2011

3. Baba T, Houkin K, Kuroda S: Novel epidemiological features of moyamoya disease. J Neurol Neurosurg Psychiatry 79:900904, 2008

4. Cheung AH, Lam AK, Ho WW, Tsang CP, Tsang AC, Lee R, Lui WM, Leung GK: Surgical outcome for Moyamoya disease - clinical and perfusion computed tomography correlation. World Neurosurg 98:81-88, 2017 
5. Cho WS, Chung YS, Kim JE, Jeon JP, Son YJ, Bang JS, Kang HS, Sohn $\mathrm{CH}$, Oh CW: The natural clinical course of hemodynamically stable adult moyamoya disease. J Neurosurg 122:82-89, 2015

6. Egashira Y, Yamauchi K, Enomoto Y, Nakayama N, Yoshimura $\mathrm{S}$, Iwama T: Disruption of cortical arterial network is associated with the severity of transient neurological events after direct bypass surgery in adult moyamoya disease. World Neurosurg 100:311-315,2017

7. Esposito G, Kronenburg A, Fierstra J, Braun KP, Klijn CJ, van der Zwan A, et al: "STA-MCA bypass with encephalo-duromyo-synangiosis combined with bifrontal encephalo-duroperiosteal-synangiosis" as a one-staged revascularization strategy for pediatric moyamoya vasculopathy. Childs Nerv Syst 31:765-772, 2015

8. Fujimura M, Bang OY, Kim JS: Moyamoya disease. Front Neurol Neurosci 40:204-220, 2016

9. Fujimura $\mathrm{M}$, Mugikura $\mathrm{S}$, Kaneta $\mathrm{T}$, Shimizu $\mathrm{H}$, Tominaga $\mathrm{T}$ : Incidence and risk factors for symptomatic cerebral hyperperfusion after superficial temporal artery-middle cerebral artery anastomosis in patients with moyamoya disease. Surg Neurol 71:442-447, 2009

10. Fujimura $\mathrm{M}$, Tominaga $\mathrm{T}$ : Current status of revascularization surgery for Moyamoya disease: Special consideration for its 'internal carotid-external carotid (IC-EC) conversion' as the physiological reorganization system. Tohoku J Exp Med 236:45-53, 2015

11. Gross BA, Du R: The natural history of moyamoya in a North American adult cohort. J Clin Neurosci 20:44-48, 2013

12. Hayashi K, Horie N, Izumo T, Nagata I: A nationwide survey on unilateral Moyamoya Disease in Japan. Clin Neurol Neurosurg 124:1-5, 2014

13. Hu J, Luo J, Chen Q: The susceptibility pathogenesis of the moyamoya disease. World Neurosurg 101:731-741,2017

14. Ilkhchoui Y, Panikkath PV, Martin H: Moyamoya disease, revascularisation surgery and anaesthetic considerations. BMJ Case Rep 2014, 2014

15. Imai H, Miyawaki S, Ono H, Nakatomi H, Yoshimoto Y, Saito $\mathrm{N}$ : The importance of encephalo-myo-synangiosis in surgical revascularization strategies for Moyamoya Disease in children and adults. World Neurosurg 83:691-699, 2015

16. Khalesi M, Pezeshki Rad M, Hamedi A, Aelami MH: Moyamoya disease mimicking encephalitis. Iran J Med Sci 39:487-490, 2014

17. Kim JS: Moyamoya disease: Epidemiology, clinical features, and diagnosis. J Stroke 18:2-11, 2016

18. Kuriyama S, Kusaka Y, Fujimura M, Wakai K, Tamakoshi A, Hashimoto S, Tsuji I, Inaba Y, Yoshimoto T: Prevalence and clinicoepidemiological features of moyamoya disease in Japan: Findings from a nationwide epidemiological survey. Stroke 39:42-47, 2008

19. Liu L, Su SW, Sun HY: Safety of extracranial-intracranial arterial bypass in the treatment of Moyamoya disease. $J$ Craniofac Surg 28(6):e522-527,2017
20. Liu XJ, Zhang D, Wang S, Zhao YL, Teo M, Wang R, Cao Y, Ye $X$, Kang S, Zhao JZ: Clinical features and long-term outcomes of Moyamoya disease: A single-center experience with 528 cases in China. J Neurosurg 122:392-399, 2015

21. Nagaraja D, Verma A, Taly AB, Kumar MV, Jayakumar PN: Cerebrovascular disease in children. Acta Neurol Scand 90:251-255, 1994

22. Pandey P, Steinberg GK: Outcome of repeat revascularization surgery for Moyamoya disease after an unsuccessful indirect revascularization. Clinical article. J Neurosurg 115:328-336, 2011

23. Rahmanian A, Ghaffarpasand F, Derakhshan N: Surgical outcome of patients with very small intracranial aneurysms; A single center experience from Southern Iran. World Neurosurg 98:470-478, 2016

24. Rahmanian A, Jamali M, Razmkon A, Kivelev J, Romani R, Alibai EA, Hernesniemi J: Benefits of early aneurysm surgery: Southern Iran experience. Surg Neurol Int 3:156, 2012

25. Rahmanian A, Seifzadeh B, Razmkon A, Petramfar P, Kivelev J, Alibai EA, Hernesniemi J: Outcome of decompressive craniectomy in comparison to nonsurgical treatment in patients with malignant MCA infarction. Springerplus 3:115, 2014

26. Research Committee on the Pathology and Treatment of Spontaneous Occlusion of the Circle of Willis; Health Labour Sciences Research Grant for Research on Measures for Intractable Diseases: Guidelines for diagnosis and treatment of Moyamoya Disease (spontaneous occlusion of the circle of Willis). Neurol Med Chir (Tokyo) 52:245-266, 2012

27. Samadian M, Alavi E, Bakhtevari MH, Rezaei O: Surgical management of giant basilar tip aneurysm associated with Moyamoya disease: A case report and literature review. World Neurosurg 84:865.e867-811, 2015

28. Starke RM, Komotar RJ, Hickman ZL, Paz YE, Pugliese AG, Otten ML, Garrett MC, Elkind MS, Marshall RS, Festa JR, Meyers PM, Connolly ES Jr: Clinical features, surgical treatment, and long-term outcome in adult patients with Moyamoya disease. Clinical article. J Neurosurg 111:936-942, 2009

29. Suzuki J, Takaku A: Cerebrovascular "moyamoya" disease. Disease showing abnormal net-like vessels in base of brain. Arch Neurol 20:288-299, 1969

30. Takeuchi K, Shimizu K: Hypoplasia of the bilateral internal carotid arteries. Brain Nerve 9:37-43, 1957

31. Yonekawa Y, Ogata N, Kaku Y, Taub E, Imhof HG: Moyamoya disease in Europe, past and present status. Clin Neurol Neurosurg 99 Suppl 2:S58-60, 1997

32. Zhang Q, Wang R, Liu Y, Zhang Y, Wang S, Cao Y, Zhao $Y$, Liu X, Wang J, Deng X, Gao F, Yang Z, Zhao M, Ge P, Ma Y, Zhao J, Zhang D: Clinical features and long-term outcomes of unilateral Moyamoya disease. World Neurosurg 96:474-482, 2016 